\title{
Coupling sections, emittance growth, and drift compensation in the use of bent solenoids as beam transport elements
}

\author{
J. Norem \\ HEP Division, Argonne National Laboratory, Argonne, Illinois 60439
}

(Received 18 November 1998; published 25 May 1999)

\begin{abstract}
Bent solenoids can transmit charged particle beams while providing momentum dispersion. While less familiar than quadrupole and dipole systems, bent solenoids can produce superficially simple transport lines and large acceptance spectrometers for use at low energies. Design issues such as drift compensation and coupling sections between straight and bent solenoids are identified, and aberrations such as shears produced by perpendicular error fields are discussed. Examples are considered which provide the basis for the design of emittance exchange elements for the cooling system of a muon collider. [S1098-4402(99)00043-9]
\end{abstract}

PACS numbers: 29.27.Eg, 29.27.Fh, 29.30.-h

\section{INTRODUCTION}

Solenoidal focusing systems have been used in many low energy, linear beam transport applications for many years [1]. High energy optical systems which will use solenoids with bends are also being considered for transport and cooling of muons in a muon collider [2,3], transport of muons for the study of muon decays [4,5], charge separation [6], and electron cooling of high energy proton and antiproton beams [7].

One of the basic assumptions generally made in high energy beam optics is that $\mathbf{B}$ fields are perpendicular to the direction of motion. Solenoids, which have a large parallel component of the magnetic field, $B_{\|}$, tend to be used more for low energy beams, since the focusing power $K$ of solenoids goes like $K \sim 1 / f \sim p^{-2}$, where $f$ and $p$ are the focal length of the solenoid section and the momentum of the particles. Straight solenoids also have the advantage that they can have large apertures, and are homogeneous and simple. Cases where the beam and the fields are roughly parallel have been worked out in detail in plasma physics examples [8].

Beam optics in straight solenoids is simple and well understood in the context of accelerator beams [1]. The optics of orbits in toroids is also well understood, since toroidal magnetic fields are used in confining plasmas. The dynamics of particle orbits in bent (toroidal) geometries, which show a variety of effects such as cross field drifts, are clearly described in Ref. [8]. Cross field drifts are due to two effects: centrifugal drift and gradB drift. Centrifugal drift is caused by the rigidity of the beam pushing particles to larger bend radii than those followed by the magnetic field lines. Grad B drifts are produced by variations in the magnetic field causing the radius of the Larmor orbit to change as the particle circles the field line. The use of external magnetic fields as well as fields from the beam itself are also common. These effects have been described in the context of plasma confinement systems such as the tokamak and the stellarator. The solutions applicable in plasma physics, however, which involve rotating the whole assembly of particles on its axis, are not generally applicable to high energy beam optics.

The use of both straight and bent elements together is not common, although these systems can do many of the things normally associated with magnetic spectrometers, such as magnetic analysis, charge separation, and transverse projections of longitudinal emittance. The magnetic fields in the transition region between the straight and bend are difficult to calculate and are dependent on physical coil geometry as well as the parameters of the bent and straight sections. Even in simple cases the magnetic field in the transition region can best be calculated numerically. When solenoids with large coils are constrained to follow bends with a small or variable radius, there are further complications and the fields and the effects they produce become even more difficult to evaluate.

This paper attempts to describe the scope of beam optics of bent solenoids, producing algorithms to evaluate aberrations and describing methods to minimize these problems. Considering the superficial simplicity of the problem, it is surprising that there are so many possible variables and effects that can be produced. Since the effects can at first seem nonintuitive, they are described systematically, with ray tracing examples using the code GPT, written by Pulsar Physics [9]. Three-dimensional numerical analysis was used because this method eliminated assumptions. While a deductive exposition starting with $d \mathbf{p} / d t=q \mathbf{v} \times \mathbf{B}$ would be valuable, there is a large range of variables and valid solutions, and this paper is intended to describe the beam effects in a more descriptive way. The approach here has been to produce accurate numerical calculations of the fields and orbits from the geometry of coils and currents, without considering approximations or analytical models.

The focus of this paper is on developing the basic principles which can be used for the design of compact high dispersion systems for longitudinal emittance exchange and momentum measurements for experimental muon 
cooling systems [10]. Examples are shown which give the magnitude of effects in realistic cases.

\section{VARIABLES}

To a first approximation, the design of a straight solenoid requires only that coils be placed in a line, with the current $i$ circulating around the desired volume equal to $i=B / \mu_{0}$, where $i$ is the current per unit length, $B$ is the desired field, and $\mu_{0}$ is the permeability constant.

The Larmor frequency, radius, and wavelength for motion in a solenoid are

$$
\begin{gathered}
f_{L}=q B / 2 \pi \gamma m_{0}, \\
r_{L}=v_{\perp} / 2 \pi f_{L}=\gamma m_{0} \beta_{\perp} c / q B=(B \rho)_{\perp} / B, \\
\lambda_{L}=\beta c / f_{L}=2 \pi \gamma m_{0} \beta c / q B=2 \pi B \rho / B,
\end{gathered}
$$

where $c$ is the speed of light, $f_{L}$ is the Larmor frequency, $m_{0}$ is the rest mass of the muon, and $q$ is its charge. The magnetic rigidity, $B \rho$ in $\mathrm{Tm}$, is equal to $p / 0.3$, where the momentum $p$ is in $\mathrm{GeV} / c$. The Larmor oscillations (which are helical) are similar to betatron oscillations (which are sinusoidal and planar) in a coordinate system rotating at $f_{L} / 2$, with the beta function equal to $\beta_{\perp}=$ $2 B \rho / B$, in the special case when all orbits pass through the axis. This paper assumes the magnet dimensions are at least a few $\lambda_{L}$ along the beam direction.

For a bent solenoid the same relations must still apply, in the limit of paraxial orbits. One nevertheless needs to be concerned with the details of the cross field drift in the bends. In addition, accurate calculation of fields and orbits in the transition between the straight and curved sections is important since the mismatch in the orbits in the two sections will tend to excite Larmor oscillations around what would be the equilibrium orbit in the next section. Thus, the basic geometry of a bent solenoid system is determined by (1) the parameters of the system (B field, coil radius $r$, bend radius $R$, and bend angle $\Theta)$, (2) the method used to couple the bent section to a straight solenoid, and (3) the correction fields used and the method of providing them. An example is the bent solenoid shown in Fig. 1 in Cartesian coordinates. In most cases presented in this paper the coil radius $r=0.3 \mathrm{~m}$, the bend radius $R=1 \mathrm{~m}$, the bend angle is $\pi / 2$, the nominal field on axis is $5 \mathrm{~T}$, and the bend is always to the right. The actual magnitude of the magnetic field $\left(B_{x}^{2}+B_{y}^{2}+B_{z}^{2}\right)^{1 / 2}$ on the median plane of the bend shown in Fig. 1 is plotted as a function of position in the bend plane in Fig. 2.

The field in the region between straight and bend is difficult to evaluate without numerical methods. In a straight solenoid the field lines are homogeneously distributed, but in the bend the field lines are distributed according to $1 / R$, where $R$ is the radius of the bend. The transition must re-

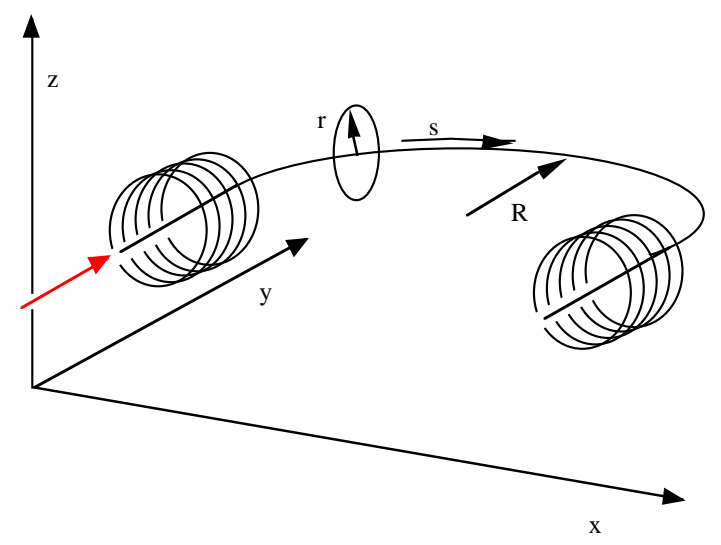

FIG. 1. (Color) The parameters of a bend which has two straight sections and no coupling sections, in Cartesian coordinates.

quire some field line redistribution in the bend plane, and except for rectangular coils, perpendicular to it.

There are a number of ways of specifying the field in the bend, depending on whether (1) the field is constant along some specified orbit, (2) the magnetic flux $\Phi=\int \mathbf{B} \cdot d \mathbf{A}$ integrated over the coil area is constant through the bend (giving no fringe field at the coupler), or (3) some other algorithm, such as the field on the coil centerline is constant. The desirability of eliminating external fields may have more weight than other constraints. The dependence of $\Phi(r / R)$ on the aspect ratio $r / R$ is shown in Fig. 3, for constant magnetic induction on axis and circular coils. In this paper the twodimensional coil elements used to produce the fields carry equal currents and the coil elements are equally spaced at their center points. The path followed by the centerlines of the current elements in the bend plane is a variable.

A plot of the radial field in the straight before the bent section shows the fields from the bend sections penetrating into the straight section, as shown in Fig. 4. These fields make tracking real beams through real bends

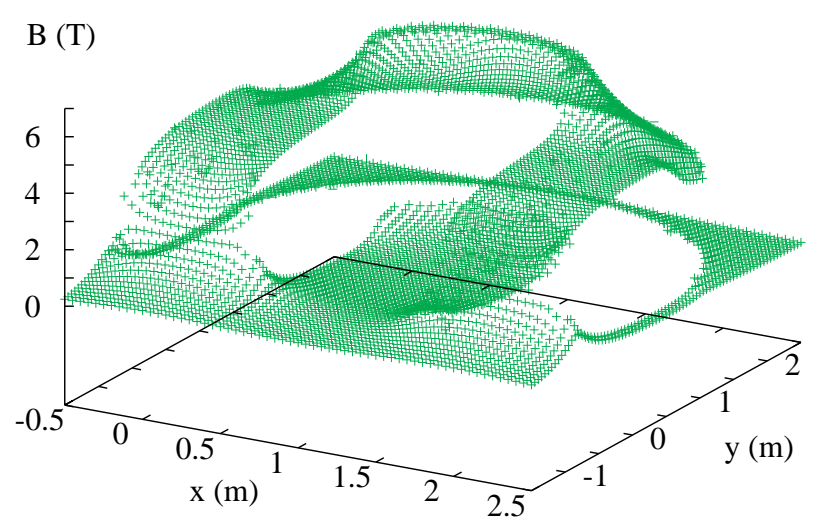

FIG. 2. (Color) A map of $|B(x, y)|$ over a bend which has two straight sections and no coupling sections, as shown in Fig. 1. 


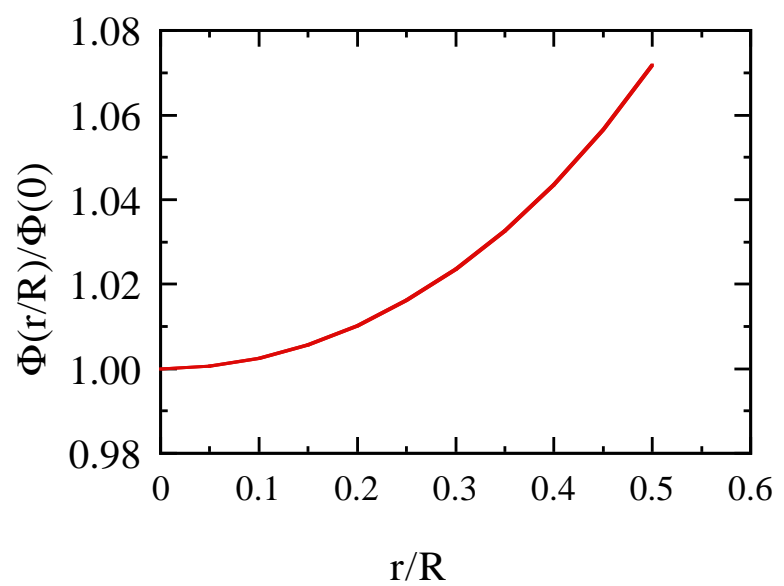

FIG. 3. (Color) Dependence of $\Phi$ on aspect ratio $r / R$, for constant axial field.

difficult and could perturb beam diagnostics located in the straight sections.

Compensating or correction fields perpendicular to the bend plane can be used to minimize cross field drifts, however, there are a number of ways of applying these fields. The beam optics depend strongly on the particular geometry used, producing rotation around the center of the beam and shears in the bend plane and perpendicular to the bend plane of the beams. These are described below.

The number of variables and options in designing a bent solenoid system is very large, and the range of effects these variables produce is not well explored.

\section{COUPLING SECTIONS AND EMITTANCE GROWTH}

The simplest example is a series of coils of radius $r$ in a straight line, where a sudden transition is made to an arc of radius $R$. This geometry is shown in Fig. 1, along with the path of a particle tracked through it, both in three dimensions and a two-dimensional projection, in Figs. 5 and 6 . The particle orbit is shown in the beam coordinate

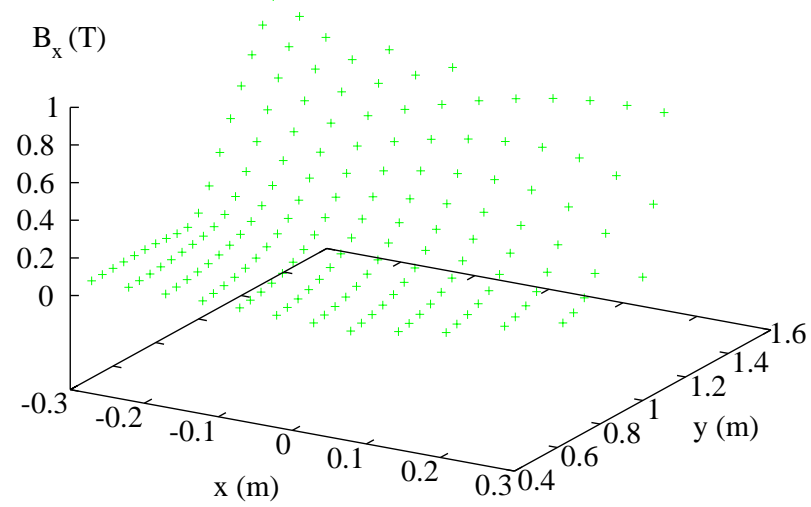

FIG. 4. (Color) Variation of $B_{x}(x, y)$, just before the bend, in Cartesian coordinates. system, where the bend is straightened out. The vertical drift is caused by two effects: centrifugal drift,

$$
\mathbf{v}_{C}=\frac{\gamma m_{0} v_{\|}^{2}}{q B^{2}} \frac{\mathbf{R} \times \mathbf{B}}{R^{2}},
$$

which depends on the components of the beam parallel to the magnetic field, and grad B drift,

$$
\mathbf{v}_{\nabla B}= \pm \frac{1}{2} v_{\perp} r_{L} \frac{\mathbf{B} \times \nabla B}{B^{2}}
$$

which depends on components of the beam velocity perpendicular to the magnetic field, and the radius of the bend [8]. Since $\nabla B / B=-\mathbf{R} / R^{2}$ and $r_{L}=\gamma m_{0} v_{\perp} / q B$, the total drift is the sum

$$
\mathbf{v}_{\text {drift }}=\mathbf{v}_{C}+\mathbf{v}_{\nabla B}=\frac{\gamma m_{0}}{q} \frac{\mathbf{R} \times \mathbf{B}}{R^{2} B^{2}}\left(v_{\|}^{2}+0.5 v_{\perp}^{2}\right) .
$$

In high energy beam transport the perpendicular components of the velocity tend to be small, $<20 \%$ of the parallel components, so the grad B drift is usually a much smaller effect than the curvature drift, i.e., $<2 \%$. This vertical drift is shown in Figs. 5 and 6 for particles initially moving parallel to the field. A particle coming into a bend section effectively begins to execute a smooth vertical drift motion around a point radially offset, by $\Delta R$, from the initial bend radius, plus a Larmor oscillation around the point of vertical drift. The sum of the two motions gives a cusp motion like a point on a circle rolling on a line with no perpendicular motion at the entrance point. The radius of the circle $r_{d}$ as well as the radial offset of the orbit $\Delta R$ is approximately equal to

$$
\Delta R \simeq r_{d} \simeq \gamma m_{0} v_{\mathrm{drift}} / q B \simeq(B \rho)_{\mathrm{drift}} / B,
$$

as shown in Ref. [1]. The coil geometry can affect these relations somewhat. Note that the angle of drift is $\phi=$ $v_{\perp} / v_{\|}=B \rho / B_{\|} R$.

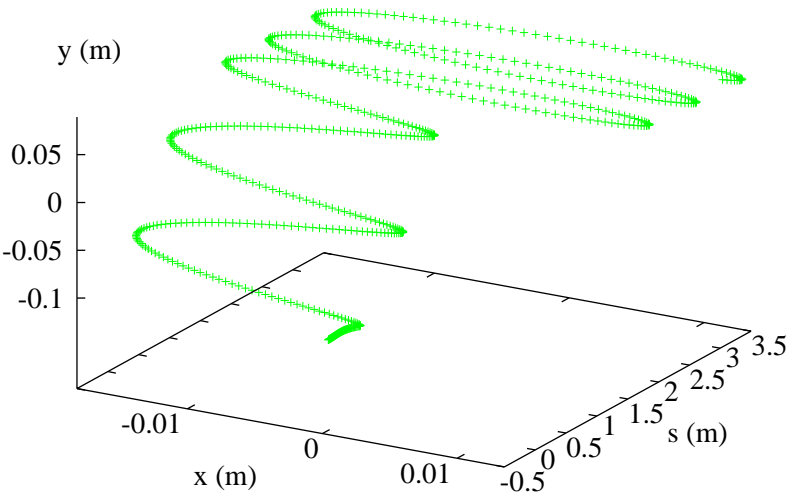

FIG. 5. (Color) Vertical drift with cusp motion in three dimensions, as a function of the length along the beam line $s$, in beam coordinates. The beam enters at the lower right. It begins to perform a vertical drift and an oscillation around a displaced equilibrium orbit. At the end of the bend, its perpendicular momentum produces oscillations around the original undisplaced horizontal position. 


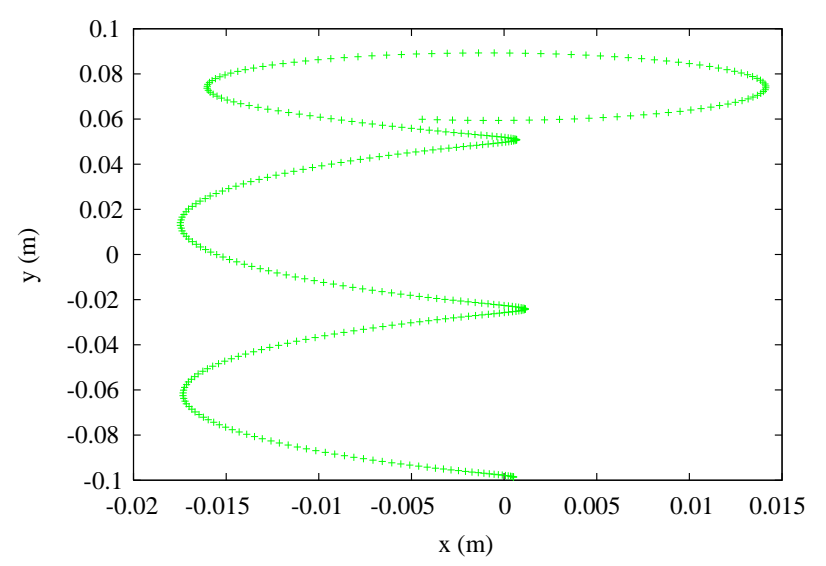

FIG. 6. (Color) Vertical drift with cusp motion from Fig. 5 in two dimensions.

At the end of the bend, the residual perpendicular momentum relative to the new (undisplaced) orbit produces oscillations around the final position. This final motion, in general, increases the angular divergence of individual orbits, and increases the emittance of a group of particles. Figure 7 shows that particles with $x^{\prime}=0$ to 0.2 have little difference in vertical drift velocities. The dominant effect for most high energy beam problems with small divergence angles is centrifugal drift. In Fig. 7, the effects of cusp orbits and drifts have been canceled by the methods outlined below to emphasize the effects of perpendicular momentum.

It is important to distinguish between two effects which can cause emittance growth when a straight and bent solenoid meet. One effect is due to the radial offset of the magnetic field lines in the bend, and is a function only of coil geometries, and the other effect is due to the centrifugal offset of the beam around the bend. Both can excite oscillations leading to emittance growth and both can be effectively eliminated by a correct coil

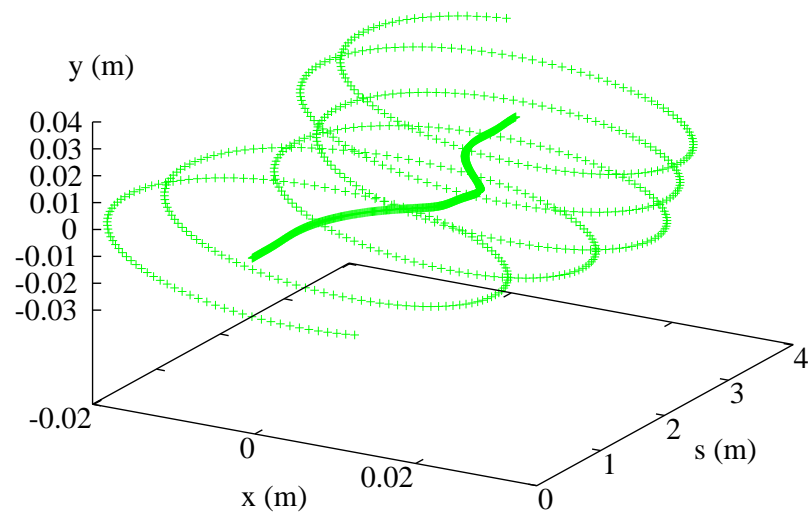

FIG. 7. (Color) The effects of perpendicular momentum, with cusp effects canceled. The particles have an initial of $x^{\prime}=0.0$ and 0.2. There is a net vertical translation of about $0.01 \mathrm{~m}$ in the large angle orbit due to grad B drift. This translation could be canceled with a bend in the opposite direction. design, at least for one momentum. The centrifugal offset can be eliminated by using compensating fields which eliminate vertical drift, and the geometrical offset can be eliminated by using couplers which are tuned to minimize the oscillations excited by the radial field offset. In the cases studied here, the centrifugal effects are larger than those caused by radial offset of the field lines.

\section{A. Simple bends, $L=n \lambda_{L}$}

The emittance of a beam is affected by the length of the bent section, since a nonintegral number of cusps will result in a residual perpendicular velocity which will result in Larmor oscillations as shown in Figs. 5 and 6. On the other hand, if the bend is exactly $L=\lambda_{L}$ long, there should be no transverse motion at the end of the cusp and it should be possible to exit the bend and couple directly to a straight section with minimal emittance growth [10]. Since the Larmor length is momentum dependent, such a bend will be optimized for only one momentum. Bends of $L=n \lambda_{L}$, for small integers $n$, should also have zero emittance growth, however, for even smaller momentum acceptances.

\section{B. Adiabatic couplers}

If space and cost were not a consideration, the solution to most of the problems associated with bent solenoids would be to have a long adiabatic transition between bent and straight sections. Figure 8 shows the effects of an adiabatic coupling section which has a final bend radius comparable to that in Fig. 6. The particle, which enters the bend near the lower right corner, slowly begins to drift upward and outward until it reaches its equilibrium offset at the upper left of the plot. The oscillations around the equilibrium at the end are a very small fraction of those which would have been present without the coupler.

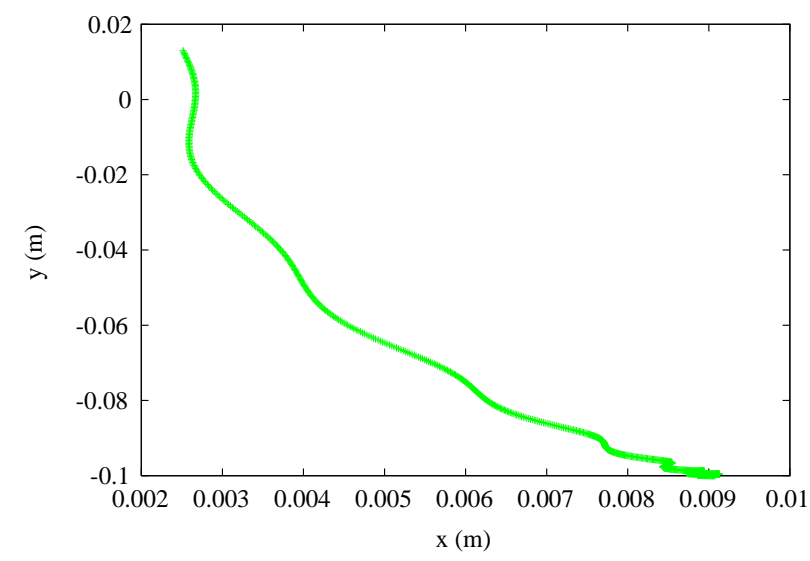

FIG. 8. (Color) Tracking through an adiabatic coupler. The particle starts at $y=-0.1 \mathrm{~m}$ and moves slowly to its equilibrium offset over many Larmor lengths without exciting large cusp oscillations. 
Adiabatic couplers can transmit a very wide momentum spectrum; however, the length of the coupler would be $n \lambda_{L}$, with $n$ large, and $\lambda_{L}$ evaluated for the highest momentum transmitted.

\section{C. $L=\lambda_{L} / 2$ couplers}

In general, coupling between straight and bent solenoids is easiest when not only the bending angle changes smoothly with distance but the first and second derivatives are also smooth. An exception to this is a special case where the cusp motion itself can be used to couple to bends of arbitrary radius.

Using the picture described in Sec. III A, it is possible to couple a straight section to a bent section with minimal residual oscillation, by using half a cusp oscillation between the straight and bent sections. Thus one can use a bend section of essentially arbitrary length and dispersion. An example of this is shown in Fig. 9. In this example, the particle again enters at the lower right and then performs one-half of a cusp oscillation, which leaves it at the equilibrium offset required to continue through the bend. However, since it is at the equilibrium offset with little perpendicular momentum it can continue propagating through the bent section with minimal oscillations.

This method, while practical in special cases, seems to be difficult to optimize, since coils of finite radii tend to introduce end effects which complicate the geometry of the transition region and can result in some residual oscillation. These small oscillations are a result of the components of $B$ perpendicular to the particle orbit which exist because the magnetic field lines do not follow the centerlines of the coils. This effect is present in all coupler geometries. It is minimized if the coil radius is

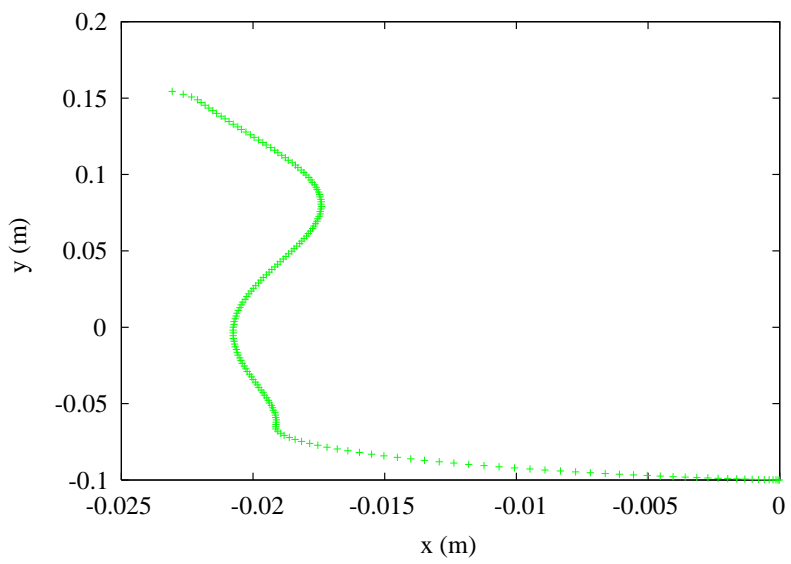

FIG. 9. (Color) An example of an orbit through a $L=\lambda / 2$ coupler with $R_{\text {coupler }} \sim 2 R_{\text {bend }}$, showing some mismatch. In this case the coil radius was $0.15 \mathrm{~m}$ and the particle exited the solenoid. Some mismatch is seen in the subsequent motion of the orbit after the coupler due to fringe fields from the straight and coupling sections. very small. The example shown in the figure was not entirely optimized and shows the effects of such a slight mismatch.

\section{Smooth $L=\lambda_{L} / 2$ couplers}

It has been shown from tracking results that discontinuities in $d \Theta / d s$ or its derivatives can excite Larmor oscillations and offsets, thus it is best if the bend starts and ends gradually. This can be accomplished by a number of options, but the smooth bend profile used by Fernow in the code ICOOL [11] has been found useful because it is simple and has smooth derivatives. To some extent this method is a combination of adiabatic and $\lambda_{L} / 2$ coupling. The expression for a bend starting at $s_{1}$ and ending at $s_{2}$ with coupling length $\sigma$ is

$$
\frac{d \Theta}{d s}=0.5\left[\tanh \left(\frac{s-s_{1}}{\sigma}\right)-\tanh \left(\frac{s-s_{2}}{\sigma}\right)\right] .
$$

The best measure of the usefulness of this curve is given by the amplitude of oscillations excited at the end of a bend. Plots of the radius of these oscillations are a realistic measure of the magnitude of emittance growth using this method. Such a graph is shown in Fig 10, which displays the radius of the Larmor oscillation excited as a function of the length $\sigma$ over which the transition is made. It is seen that the minimum at $\sigma \sim \lambda_{L} / 2$ is consistent with the arguments presented in Sec. III C.

\section{DRIFT COMPENSATION}

In order to eliminate the effect of the perpendicular drift, it is possible to introduce a vertical magnetic field equal to $B_{v}=B \rho / R$, where $B \rho$ is the rigidity of the beam and $R$ is the radius of the bend. There are a number of coil geometries which produce this field using either (1) external field coils or (2) tilting the solenoidal coils to produce a transverse component. A combination of external coils and tilted coils is also an option, and perhaps the most flexible solution.

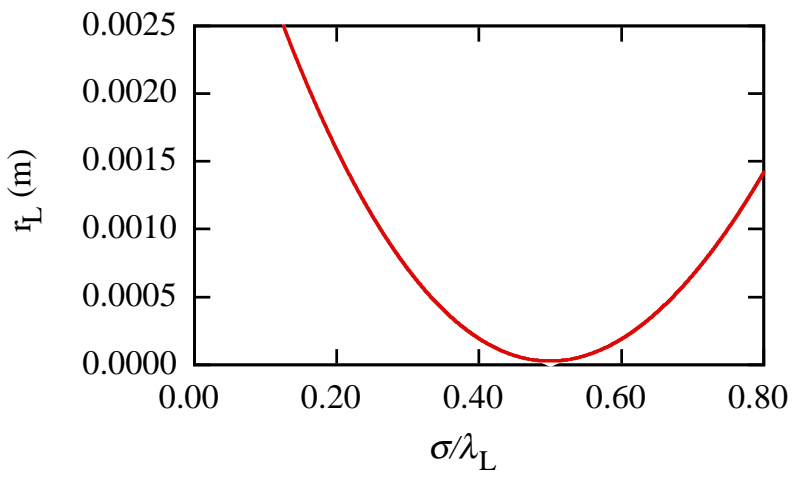

FIG. 10. (Color) The emittance growth plotted against coupling length in short, smooth couplers. 
The drift velocity in a strong $\mathbf{B}$ field is given by $\mathbf{v}_{\text {drift }}=$ $(\mathbf{F} \times \mathbf{B}) / q B^{2}$ for a force $\mathbf{F}=q\left(\mathbf{v} \times \mathbf{B}_{\perp}\right)$. Using the vector identity $(\mathbf{S} \times \mathbf{T}) \times \mathbf{U}=\mathbf{T}(\mathbf{U} \cdot \mathbf{S})-\mathbf{S}(\mathbf{U} \cdot \mathbf{T})$ with $\left(\mathbf{v} \cdot \mathbf{B}_{\perp}\right) \sim 0$ allows one to see that the drift velocity is in the direction of the perpendicular field component producing the drift, and is equal to $\mathbf{v}_{\text {drift }}=v_{\|} \mathbf{B}_{\perp} / B_{\|}$. This can be seen, for example, as compensating an upward vertical drift from a field line by pointing the field line down enough to cancel the vertical motion.

The simplest geometry for producing a uniform vertical field over a small region near the center of the solenoidal coils would be to use external coils carrying a current parallel to the solenoidal field. The density of the current should be proportional to the cosine of the angular height of the coils from the bend plane. This "cosine theta" geometry has wide application in the design of superconducting bending magnets. We have considered using external fields of this type, which would allow the vertical field to be independent of the solenoidal field. The overall configuration of a compensating field system with independent coils, considering general bend geometries, end effects, and beam perturbations, tends to be difficult to design and optimize.

\section{A. Tipped and turned coils}

It is possible to tilt the solenoidal coil elements to provide the majority of the required vertical component without external coils, as shown in Fig. 11. Note that the tilted coils produce a longitudinal component of the current which is proportional to the cosine of the angular height above the bend plane, as described above. Tilting the coils by an angle proportional to the local bend radius provides a natural transition between the straight and curved regions. The vertical bend field which can compensate the centrifugal drift is most easily provided by introducing a tip angle $\theta$ which produces a vertical component of the solenoidal field $B_{v}(x, y)$ for a particle moving down the center of the solenoid. This angle can be determined using tracking to give zero net vertical drift through a bend as shown in Figs. 12 and 13. Tracking has given a value of

$$
\theta \sim \frac{6.7 p_{[\mathrm{GeV} / c]}}{R_{[\mathrm{m}]} B_{[\mathrm{T}]}},
$$

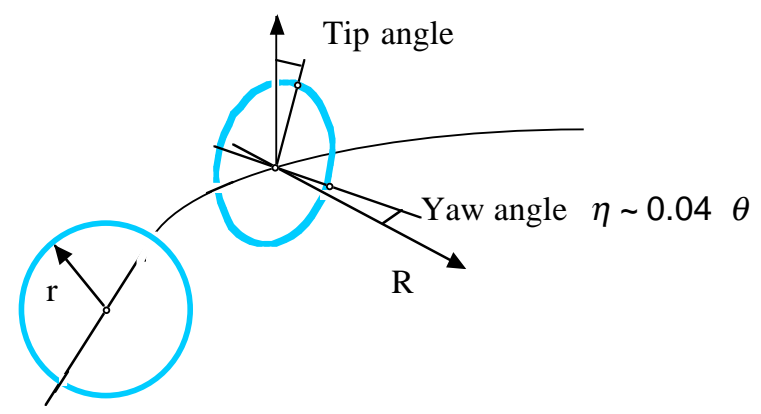

FIG. 11. (Color) The tipped and turned coils geometries. for bend radii and fields of a few $\mathrm{m}$ and $\mathrm{T}$. Since the tilt angle depends on momentum, this option fixes the central momentum of the bend. Horizontal drifts are smaller, and the yaw angle $\eta$ required for the coils to be turned is a small fraction of the tilt angle, perhaps $\eta \sim 0.04 \theta$. Numerical calculations of the fields have shown that when the coils are tipped, the vertical field produced, $B_{v}(R)$, is roughly proportional to $B_{\|}$, and thus also has a $1 / R$ dependence. Thus tipped coils give a good approximation of the ideal $B_{v}(R)$ over the full coil aperture.

In the case shown in Figs. 12 and 13, a number of details of the optics are visible. A particle enters the magnet parallel to the field and is deflected towards smaller $R$, since it tends to follow the inward motion of field lines going around the bend. A slight upward then downward motion is also seen in the coupling section, due to a slight mismatch between the required vertical field and that produced by tipping the coils. This effect could be presumably eliminated by trimming the longitudinal distribution of the vertical field by trimming tilt angles of the magnets (i.e., using a slightly increased value of $\sigma$ for the tilt angle distribution). At the exit of the magnet the field lines return to their initial distribution, and the same slight mismatch produces a downward then upward motion. A reference orbit can exit with the same $(x, y)$ coordinates it entered the bend.

\section{B. Dispersion}

A bent solenoid will produce a vertical dispersion due to the momentum dependence of the vertical drift angle. This vertical dispersion will be modified by the vertical drift introduced by the compensating field.

The vertical drift angle, including the centrifugal term, will then be

$$
\phi=v_{\perp} / v_{\|}=B \rho / B_{\|} R-B_{\perp} / B_{\|},
$$

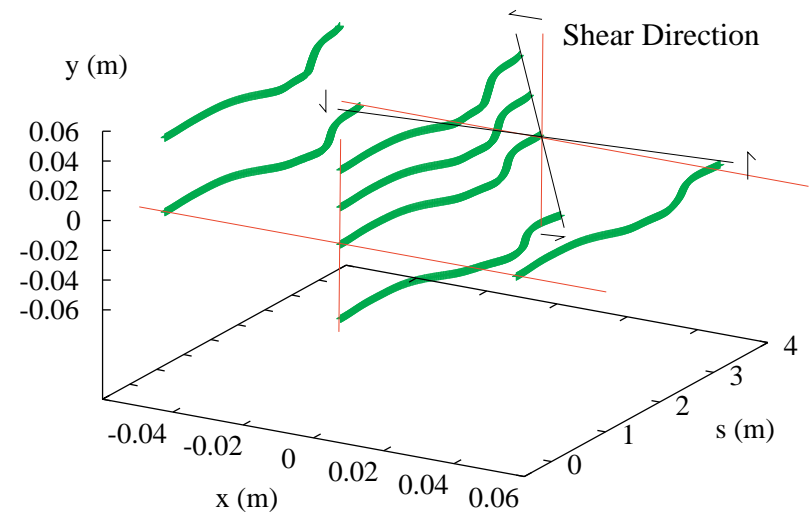

FIG. 12. (Color) Tracking through a bend in three dimensions with tipped and turned coils. The tracks show shear from perpendicular field components which is more clearly seen in Fig. 13. 


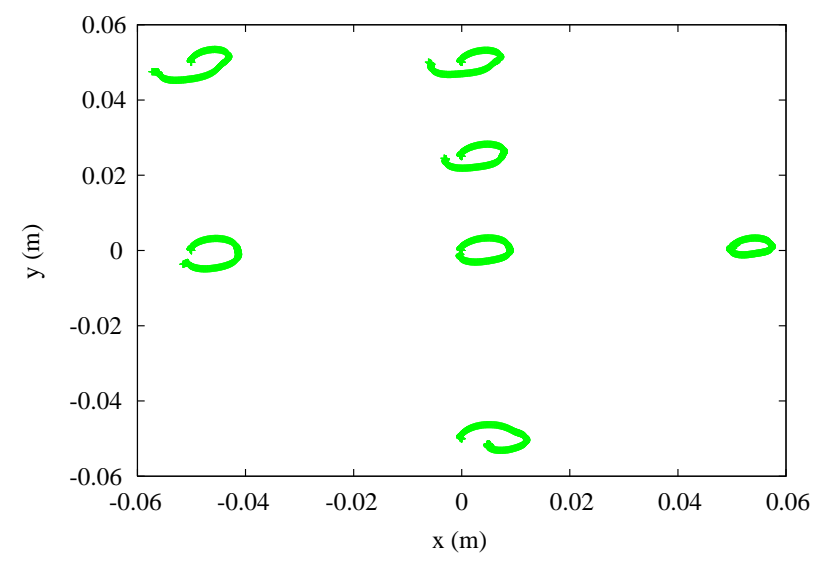

FIG. 13. (Color) A two-dimensional projection of the tracks shown in Fig. 12. The (xy) projections show a clockwise drift combined with a horizontal or vertical displacement which depends on the initial position.

and the dispersion is due only to the first term,

$$
D=\int B \rho / B_{\|} R d s \sim \Theta B \rho / B,
$$

where $\Theta$ is the total bend angle. Figure 14 shows the results of tracking a particle 1.5 times the nominal momentum shown in Figs. 12 and 13. The longer Larmor length produces some residual perpendicular velocity as described in Sec. IIID due to a slight mismatch of the coupling section.

\section{Shears}

Although particles can be transmitted through the system with small emittance growth or relative deflection, the motion of off axis particles is complex. The primary aberration associated with off axis orbits is a linear shear between the entrance and exit of a bend. This shear results in a small horizontal translation of orbits which depends on the vertical position in the solenoid, and a

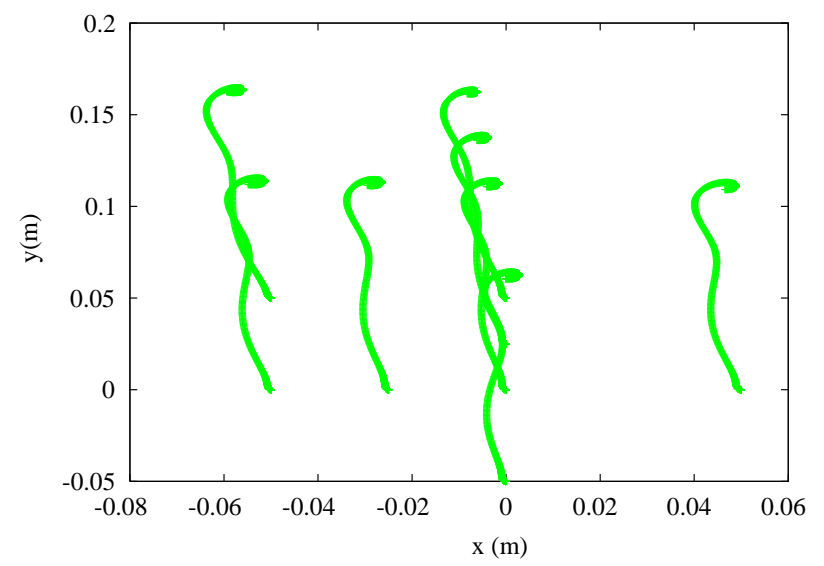

FIG. 14. (Color) Tracking a momentum 1.5 times that shown in Fig. 12 shows some mismatch due to the coupler length. vertical translation of the orbits which depends on the horizontal position in the solenoid. Tracking results from an array of particles introduced into a bend with circular coils tipped and turned to give no axial deflection, and no initial perpendicular momenta, are shown in Figs. 12 and 13. These tracks show that there is an offset between the entrance and exit positions and, for a bend angle of $\Theta=\pi / 2$ and a $5 \mathrm{~T}$ field, this offset has the form

$$
\Delta x=0.11 y \text { and } \Delta y=0.047 x,
$$

assuming that the central ray $(x, y)=(0,0)$ shows no offset. The translation seems to be linear in $x$ or $y$ and the coefficients of $x^{2}, y^{2}$, and $x y$ terms are negligible.

These shears result from undesirable components of the compensating field $\mathbf{B}_{v}(R)$ due to the physical geometry of the coil system. For tipped circular coils and a circular bend, there is a horizontal component of $\mathbf{B}_{v}(R)$ that depends on vertical position, resulting in a curvature of the vertical magnetic field lines. This curvature causes the horizontal drift/shear. The vertical drift/shear is caused by the difference between the vertical field produced and the $1 / R$ field required. These components can be modified somewhat by external coils.

Ideal beam optics requires a compensating field whose intensity is proportional to $1 / R$, with no horizontal components in the volume occupied by the beam. Unfortunately this requirement violates Ampere's Law. Integrating $\int \mathbf{B} \cdot d \mathbf{l}=\mu_{0} I$, where $I=0$ is the enclosed current, would require nonzero horizontal fields at the top and bottom if the values of $\mathbf{B}$ at two radial positions were different, as shown in Fig. 15. Thus, the sum of the magnitudes of the horizontal and vertical shears produced is an invariant for a given bend geometry, dependent on the aspect ratio of the bend $r / R$, end effects, and the coil geometry. Accurate evaluation of these aberrations must rely on calculation directly from the exact coil geometries since these effects result directly from the coil geometry.

\section{Optimizing external fields}

For circular bends, the field can be provided entirely by the tipped and turned coils or external coils can be used. For the cases described here, the length of the

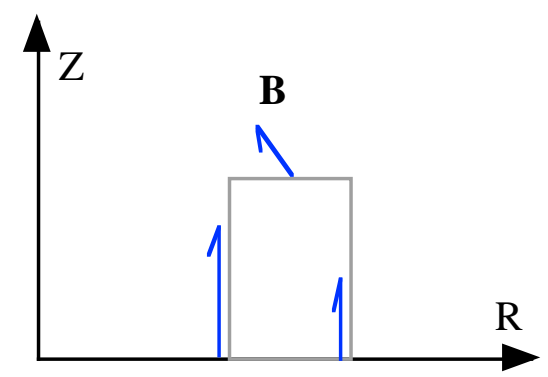

FIG. 15. (Color) The integral of $\int \mathbf{B} d \mathbf{l}$ around the dotted line must be zero if no current is carried. 
bent solenoids tends to be small multiples of the $2 \lambda_{L} / 2$ probably required for the two coupling sections.

External coils seem to provide the most flexibility. The fields required tend to be strong, and the perturbations on the beam from end effects are significant. Using tipped and turned coils may provide less of a magnetic perturbation on the beam, but it seems desirable to have some correction capability to minimize shears and provide some momentum tuning capability. This implies that some capability of generating fields from external coils is desirable.

\section{APPROXIMATIONS}

The processes outlined above imply that the primary method used to attack the problems of bent solenoids should be to use coil positions to derive fields, and then track particles through these fields. While precise, this method can be very time consuming, and the relevance of tracking simpler cases inevitably arises. The problem with using approximations is that the phenomena described here tend to be due to the strong longitudinal field, which is not present in most particle tracking problems. Small effects due to the specific positions of coils also contribute. Since these problems tend to be associated only with this class of coil geometry, the effects are likely to have somewhat nonintuitive results. Thus it seems desirable to avoid approximations until they have been carefully calibrated against precise calculations.

One example of the sensitivity of particle orbits to the magnet geometry is shown in Fig. 16, which demonstrates the results of changing the coil size from 0.3 to $0.03 \mathrm{~m}$. The particle orbit, which was optimized for the larger radius coil, produces less overall deviation in the smaller coils, presumably because less motion of the magnetic

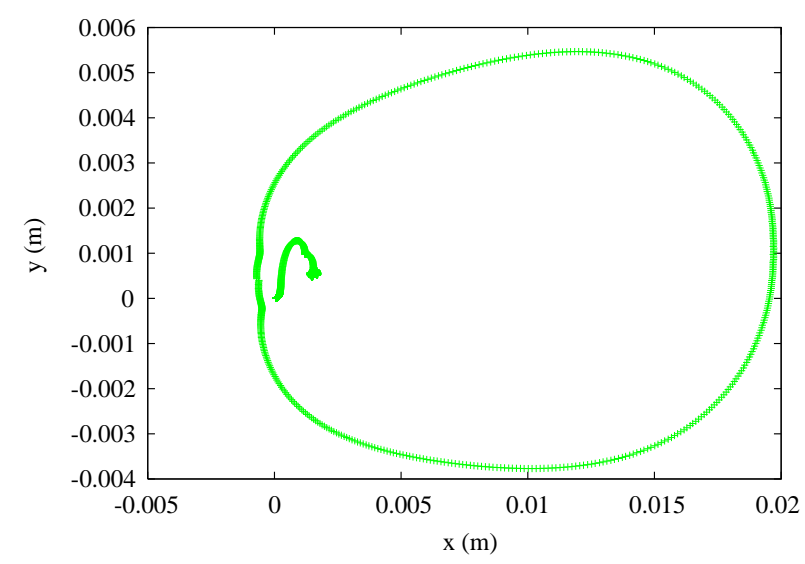

FIG. 16. (Color) Tracking through a bend with coil radii of 0.03 and $0.3 \mathrm{~m}$. The coil geometry was optimized for zero translation with coil radius $r=0.3 \mathrm{~m}$, as seen in the large loop. Reducing the coil size alters the fields, with a corresponding change in the particle orbit. This new orbit, which has not been tuned for zero translation, is quite different, with less perpendicular motion. Both tracks start at $(0,0)$. field lines is required. (The smaller radius coil set could also be optimized for zero overall deflection.)

\section{EMITTANCE EXCHANGE SECTION DESIGN}

Emittance exchange from longitudinal to transverse requires that the beam be dispersed in momentum so that wedges can then preferentially degrade the high energy beams. In general, emittance exchange will take place in more than one bend. The particular example described in Fig. 17, proposed by Palmer [2] and Fernow [11], uses short smooth couplers with $L=\lambda_{L} / 2$ to provide the maximum possible dispersion with the minimum emittance growth at the transitions. Tracking [2] has shown that transverse emittance increase is tolerable.

\section{A. Magnet issues}

The primary magnet constraint seems to be the ratio of dispersion to magnet cost, since a very wide range of parameters will satisfy the optical constraints. It is assumed that the coils are spaced densely enough so that coil ripple will be negligible. However, the coils can be quite large and bend radii can be small, giving aspect ratios $r / R$ limited primarily by coil engineering. Dispersion is limited by the fact that bends greater than $\Theta=\pi$ are difficult. Smooth $\lambda_{L} / 2$ couplers seem to provide the best combination of small emittance growth and minimum stored energy (cost), and tipped coils seem to be the simplest way of providing a compensating field, if desired. With no compensating field both charge states could be accommodated. The volume and cost of such a magnet would be increased. A variety of coil shapes could be used and these would weakly affect the optics.

\section{B. Wedge constraints}

Emittance growth must also be minimized in the energy loss process. Wedges must be distributed along the beam over a length $>\lambda_{L} / 2$. This constrains the geometry of the wedge placement. The centroid of a Larmor oscillation will be moved a distance $r_{L} \Delta p / p$ by a single absorber. Defining the vector $\mathbf{r}_{L, i} \boldsymbol{\chi}_{i}=\Delta p / p$, whose amplitude is proportional to the momentum loss in a part of the absorber, and whose phase is the phase of the Larmor oscillation (see Fig. 18), there should be minimal emittance growth if the vector sum $\Sigma_{\boldsymbol{\chi}_{i}}$ is minimized. This implies that two absorbers $L=\lambda_{L} / 2$ apart or $n$ absorbers separated by a distance $L=\lambda_{L} / n$ would be close to optimum, as would very long absorbers with a density which adiabatically rises and falls.

\section{Timing}

A particle which moves around a bend on a larger radius will fall behind another on a smaller radius by a distance $\delta s=\Delta R \Theta$, where the bend angle is $\Theta$ and the difference 


\section{LONGITUDINAL COOLING}

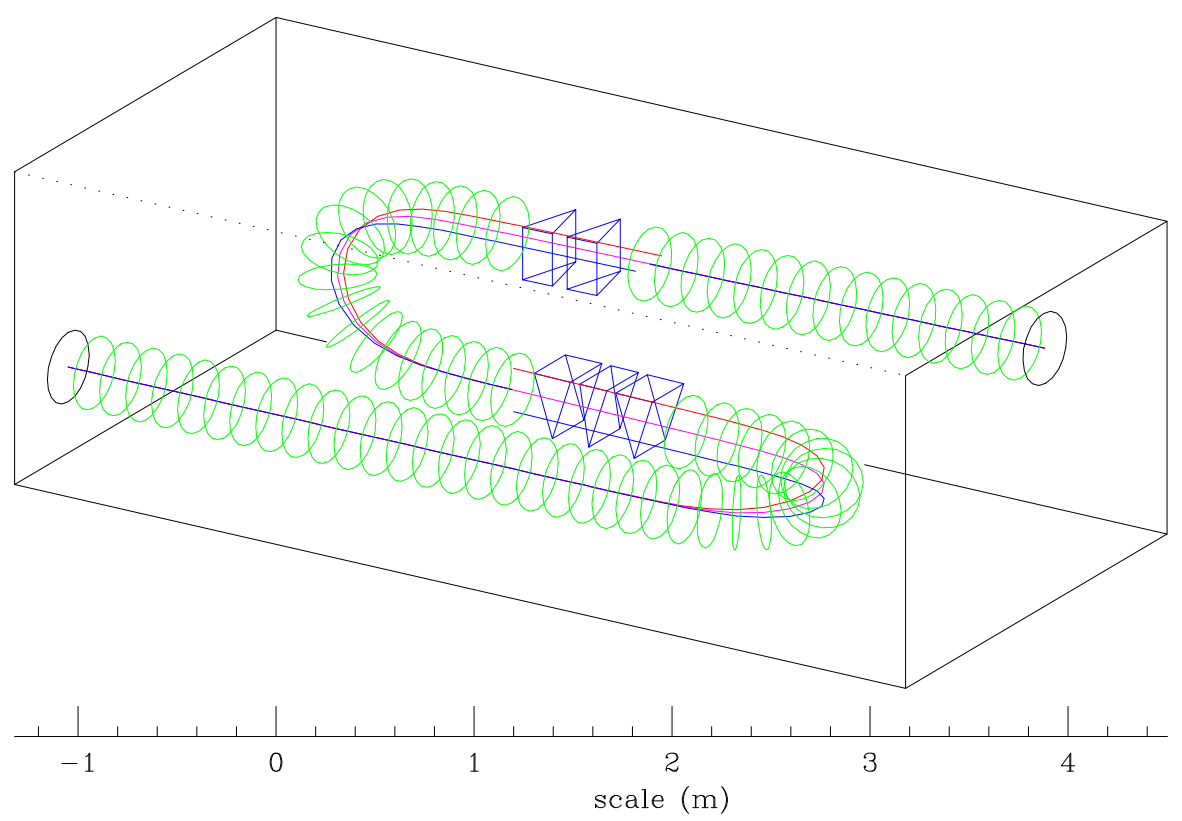

FIG. 17. (Color) One possible emittance exchange option.

in radii is $\Delta R$. The time interval generated will be $\delta t=$ $\delta s / \beta c$. If the total number of right and left, up and down bends is equal this time delay will cancel out since the magnetic flux lines are continuous through a series of bends. An additional complexity is time spread due to the difference in momenta. It is assumed that linac sections can cancel or compensate for this using sections which have a synchrotron phase advance of $\pi$ radians, which will slow down fast particles and speed up slow ones.

\section{Momentum acceptance}

The momentum acceptance of the bend system will be limited by the magnet aperture and the tolerable emittance increase. Mismatches become somewhat more difficult at higher momenta, due to the longer Larmor length. Nevertheless, designing large acceptance, high dispersion, systems with comparatively small emittance growth should proceed from the design of bends and coupling sections which produce minimum changes in the position and perpendicular momentum of particles through the bend.

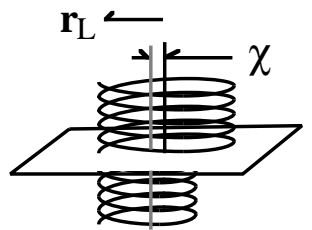

FIG. 18. The translation of the centroid of Larmor oscillations in an absorber.

\section{CONCLUSIONS}

Although superficially simple, optimization of bent solenoid systems can be difficult because of their nonlinear behavior and the lack of symmetry. This makes the use of approximate solutions and simplifying assumptions difficult, in what, for most, can be a nonintuitive environment.

While the basic beam optics of bent solenoids is fairly simple, depending on the dimensions of the coils and the bends, it is important to optimize coupling sections between straights and bends, use appropriate fields for drift compensation, and understand aberrations that may result from the completed system. Tracking programs which use coil positions and currents as input give the most complete and reliable solutions to these problems, and their input data is also useful to those who would actually construct such a coil system.

\section{ACKNOWLEDGMENTS}

This paper has profited from suggestions made during discussions with R. Palmer and R. Fernow of BNL, J.-F. Ostiguy of FNAL, K. McDonald of Princeton, S. Berg and G. Hanson of Indiana University, and M. Berz of Michigan State University. This work was supported by the U.S. Department of Energy.

[1] S. Humphries, Charged Particle Beams (Wiley, New York, 1990). 
[2] C. M. Ankenbrandt et al., Phys. Rev. ST Accel. Beams (to be published).

[3] J. C. Gallardo, R. C. Fernow, and R. B. Palmer, in Physics Potential and Development of $\mu-\mu$ Colliders, edited by D. Cline, AIP Conf. Proc. No. 441 (AIP, New York, 1998), p. 282.

[4] W. Molzon, in Workshop on Physics at the First Muon Collider and at the Front End of a Muon Collider, edited by S. Geer and R. Raja, AIP Conf. Proc. No. 435 (AIP, New York, 1998), p. 152.

[5] M. Bachman, in Workshop on Physics at the First Muon Collider and at the Front End of a Muon Collider, edited by S. Geer and R. Raja, AIP Conf. Proc. No. 435 (AIP, New York, 1998), p. 460.
[6] R. Noble (private communication).

[7] P.P. Bagley et al., 1996 DPF/DPB Summer Study on New Directions for High Energy Physic (Stanford Linear Accelerator Center, Menlo Park, CA, 1997).

[8] F. F. Chen, Introduction to Plasma Physics (Plenum, New York, 1974)

[9] Pulsar Physics, De Bongerd, NL-3762 XA Soest, The Netherlands, 1998, http://www.pulsar.nl.

[10] C. Lu, K. T. McDonald, and E. J. Prebys, Princeton University Technical Note Princeton/ $\mu \mu / 97-8$.

[11] R. Fernow, ICOOL: A Fortran Program to Simulate Muon Ionization Cooling (unpublished); http://pubweb. bnl.gov/people/fernow/readme.html. 